\title{
Editorial: Facing the Upcoming of Multidrug-Resistant and Extensively Drug-Resistant Bacteria: Novel Antimicrobial Therapies (NATs)
}

\author{
Angel León-Buitimea ${ }^{1,2 *}$, Jose Ruben Morones-Ramírez ${ }^{1,2}$, Jason H. Yang ${ }^{3,4}$ and \\ Rafael Peña-Miller ${ }^{5}$ \\ ${ }^{1}$ Facultad de Ciencias Químicas, Universidad Autónoma de Nuevo León, UANL, San Nicolás de los Garza, Mexico, ${ }^{2}$ Centro \\ de Investigación en Biotecnología y Nanotecnología, Facultad de Ciencias Químicas, Universidad Autónoma de Nuevo León, \\ Parque de Investigación e Innovación Tecnológica, Apodaca, Mexico, ${ }^{3}$ Center for Emerging and Re-Emerging Pathogens, \\ Rutgers New Jersey Medical School, Newark, NJ, United States, ${ }^{4}$ Department of Microbiology, Biochemistry and Molecular \\ Genetics, Rutgers New Jersey Medical School, Newark, NJ, United States, ${ }^{5}$ Center for Genomic Sciences, Universidad \\ Nacional Autónoma de México, Cuernavaca, Mexico
}

Keywords: antibiotic resistance, multidrug-resistant bacteria, extensively drug-resistant bacteria, antimicrobial therapies, combinatorial treatments, metal nanoparticles, antimicrobial peptides

\section{Editorial on the Research Topic}

Facing the Upcoming of Multidrug-Resistant and Extensively Drug-Resistant Bacteria: Novel Antimicrobial Therapies (NATs)

Edited and reviewed by: Jean Marie François, Institut Biotechnologique de Toulouse (INSA), France

*Correspondence:

Angel León-Buitimea angelxlogan@gmail.com

Specialty section: This article was submitted to Synthetic Biology,

a section of the journal Frontiers in Bioengineering and Biotechnology

Received: 01 December 2020 Accepted: 04 February 2021 Published: 23 February 2021

Citation:

León-Buitimea A, Morones-Ramírez JR, Yang $\mathrm{JH}$ and Peña-Miller R (2021) Editorial: Facing the Upcoming of Multidrug-Resistant and Extensively Drug-Resistant

Bacteria: Novel Antimicrobial Therapies (NATS).

Front. Bioeng. Biotechnol. 9:636278. doi: $10.3389 /$ fbioe. 2021.636278
Antimicrobial resistance is one of the largest looming threats to global health (Friedman et al., 2016), increasing the morbidity and mortality associated with bacterial infections (Ventola, 2015). ESKAPE pathogens (Enterococcus faecium, Staphylococcus aureus, Klebsiella pneumoniae, Acinetobacter baumannii, Pseudomonas aeruginosa, and Enterobacter spp.) are responsible for the majority of nosocomial infections (Ma et al., 2020) and commonly "escape" the biocidal action of antimicrobial agents (Mulani et al., 2019). The frequent and increasing use of antibiotics in medical practice drives the emergence of multidrug-resistant (MDR) and extensively drug-resistant (XDR) pathogens (Magiorakos et al., 2012; Prestinaci et al., 2015). There is an urgent and critical need to design and engineer novel therapeutic alternatives for eradicating MDR and XDR bacteria through burgeoning technologies such as metal nanoparticles, genetic engineering, synthetic biology, peptide therapeutics, and combinatorial treatments. In this Research Topic, we assemble ten original articles highlighting recent discoveries around Novel Antimicrobial Therapies (NATs) against MDR and XDR bacteria.

Seven original research articles spanning diverse disciplines describe the development of NATs for clinically-relevant MDR pathogens. One study describes the one-pot synthesis of Ag- $\mathrm{ZnO}$ nanoparticles at low temperatures and demonstrated remarkable antimicrobial activity of these nanoparticles against methicillin-resistant Staphylococcus aureus (MRSA) (Naskar et al.). Another study achieved successful phytomedited synthesis of green $\mathrm{TiO}_{2} \mathrm{NPs}$ that proved to be effective for treating biofilm-based bacterial and fungal infections (Al-Shabib et al.).

Another research article assessed the therapeutic efficacy of antimicrobial combinations on carbapenemase-producing Enterobacterales (CPE). The authors showed how antimicrobial combinations synergized against most CPE expressing resistance genes. These antimicrobial combinations may facilitate the successful treatment of patients infected with CPE (Zhou et al.). Another original research article identified two potent combinations of antibiotics for clinical MRSA infection, both in vitro and in vivo (Yu et al.). A separate study found that the compound 
2,4-Di-Tert-Butylphenol isolated from an endophytic fungus substantially reduced the secretion of virulence factors and biofilm and its associated factors controlled by quorum sensing in a dose-dependent manner in Pseudomonas aeruginosa (Mishra et al.).

Furthermore, a study tested the anti-virulence activity of potential uridine diphosphate glucose pyrophosphorylase (UDPG:PP) inhibitors and showed that these inhibitors are a potential drug candidates against Streptococcus pneumoniae infections (Cools et al.). New putative antimicrobial candidates were reported by Okella et al. They designed an antimicrobial peptide and performed target identification based on a putative antimicrobial peptide motif derived from fish. From all the peptide motifs generated in this work, the authors identified Pleurocidin (secreted by flatfish) as having strong antimicrobial potential.

Three review articles included in this special issue address the use of NATs to face MDR bacteria. A mini-review discusses combination treatments (particularly antimicrobial peptides and metal nanoparticles) as a pathway to develop antimicrobial therapeutics with broad-spectrum antibacterial action, bactericidal instead of bacteriostatic activity, and better efficacy against MDR bacteria (León-Buitimea et al.). Another review explored the possibility of designing antimicrobial nanoparticlebased devices to exploit the potential of antimicrobial nanoparticles to combat MDR pathogens (Gómez-Núñez et al.). Finally, a third review describes the mechanisms associated with drug resistance in pyogenic streptococci and discusses the advantages and limitations of innovative therapeutic strategies

\section{REFERENCES}

Friedman, N. D., Temkin, E., and Carmeli, Y. (2016). The negative impact of antibiotic resistance. Clin. Microbiol. Infect. 22, 416-422. doi: 10.1016/j.cmi.2015.12.002

Ma, Y. X., Wang, C. Y., Li, Y. Y., Li, J., Wan, Q. Q., Chen, J. H., et al. (2020). Considerations and caveats in combating ESKAPE pathogens against nosocomial infections. Adv. Sci. 7:1901872. doi: 10.1002/advs.201901872

Magiorakos, A. P., Srinivasan, A., Carey, R. B., Carmeli, Y., Falagas, M. E., Giske, C. G., et al. (2012). Multidrug-resistant, extensively drug-resistant and pandrug-resistant bacteria: An international expert proposal for interim standard definitions for acquired resistance. Clin. Microbiol. Infect. 18, 268-281. doi: 10.1111/j.1469-0691.2011.03570.x

Mulani, M. S., Kamble, E. E., Kumkar, S. N., Tawre, M. S., and Pardesi, K. R. (2019). Emerging strategies to combat ESKAPE pathogens in the era of antimicrobial resistance: a review. Front. Microbiol. 10:539. doi: 10.3389/fmicb.2019. 00539 such as bacteriocins, bacteriophage, phage lysins, and metal nanoparticles (Alves-Barroco et al.).

In summary, this group of articles contributes to the search for new therapeutic strategies to combat antibacterial resistance. MDR and XDR infections are growing in incidence; the main challenges facing society are now to design, develop, and evaluate new therapeutic strategies that can spearhead the development of alternative therapies against clinically-relevant MDR pathogens.

\section{AUTHOR CONTRIBUTIONS}

All authors listed have made a substantial, direct and intellectual contribution to the work, and approved it for publication.

\section{FUNDING}

This work was supported by funding from the National Institutes of Health, grant R00GM118907 to JY, the Universidad Autónoma de Nuevo León (Paicyt 2016-2017, Paicyt 2019-2020, and Paicyt 2020-2021), and CONACyT Science Grants (Basic Science grant 221332, Fronteras de la Ciencia grant 1502, and Infraestructura Grant 279957) to JM-R. AL-B was supported by Beca de Posdoctorado Nacional 2018-2020.

\section{ACKNOWLEDGMENTS}

We thank the contributing authors for submissions and the reviewers for their time. We also thank Dr. Rustam Aminov for handling this Research Topic.
Prestinaci, F., Pezzotti, P., and Pantosti, A. (2015). Antimicrobial resistance: a global multifaceted phenomenon. Pathog. Glob. Health 109, 309-318. doi: 10.1179/2047773215Y.0000000030

Ventola, C. L. (2015). The antibiotic resistance crisis: part 1: causes and threats. $P$ T 40, 277-283.

Conflict of Interest: The authors declare that the research was conducted in the absence of any commercial or financial relationships that could be construed as a potential conflict of interest.

Copyright (C) 2021 León-Buitimea, Morones-Ramírez, Yang and Peña-Miller. This is an open-access article distributed under the terms of the Creative Commons Attribution License (CC BY). The use, distribution or reproduction in other forums is permitted, provided the original author(s) and the copyright owner(s) are credited and that the original publication in this journal is cited, in accordance with accepted academic practice. No use, distribution or reproduction is permitted which does not comply with these terms. 